\title{
Grasping the invisible: Semantic processing of abstract words
}

\author{
Lenka Zdrazilova $\cdot$ Penny M. Pexman
}

Published online: 17 May 2013

(C) Psychonomic Society, Inc. 2013

\begin{abstract}
The problem of how abstract word meanings are represented has been a challenging one. In the present study, we extended the semantic richness approach (e.g., Yap, Tan, Pexman, \& Hargreaves in Psychonomic Bulletin \& Review 18:742-750, 2011) to abstract words, examining the effects of six semantic richness variables on lexical-semantic processing for 207 abstract nouns. The candidate richness dimensions were context availability (CA), sensory experience rating (SER), valence, arousal, semantic neighborhood (SN), and number of associates (NoA). The behavioral tasks were lexical decision (LDT) and semantic categorization (SCT). Our results showed that the semantic richness variables were significantly related to both LDT and SCT latencies, even after lexical and orthographic factors were controlled. The patterns of richness effects varied across tasks, with CA effects in the LDT, and SER and valence effects in the SCT. These results provide new insight into how abstract meanings may be grounded, and are consistent with a dynamic, multidimensional framework for semantic processing.
\end{abstract}

Keywords Word meaning · Visual word recognition . Semantic memory

In recent years, much progress has been made in understanding how word meaning is retrieved. One fruitful approach has been to examine the effects of semantic richness in lexical and semantic processing (for a review, see Pexman, 2012). That is, a number of theories now attempt to describe semantic representation. These models make different claims about the basic units or dimensions of semantic information; for instance, meaning can be derived from lexical co-occurrence information (how words are used

L. Zdrazilova $\cdot$ P. M. Pexman

University of Calgary, Calgary, Alberta, Canada

P. M. Pexman $(\bowtie)$

Department of Psychology, University of Calgary, 2500 University

Drive NW Calgary, Alberta T2N1A3, Canada

e-mail: pexman@ucalgary.ca in language; e.g., Burgess \& Lund, 2000), semantic features (attributes derived from experience with concepts; e.g., McRae, Cree, Seidenberg, \& McNorgan, 2005), or sensorimotor experience (grounded in perceptual representations; e.g., Barsalou, 1999). Support for each of these frameworks has been provided by studies showing that each of these dimensions is related to lexical-semantic processing. That is, lexical-semantic processing is facilitated for words with more associates (number-of-associates effects; Duñabeitia, Avilés, \& Carreiras, 2008) or that occur in contexts similar to those of many other words (semantic neighborhood effects; Buchanan, Westbury, \& Burgess, 2001), or that generate many semantic features (number-of-features effects; Grondin, Lupker, \& McRae, 2009; Pexman, Lupker, \& Hino, 2002), or that refer to concepts that are easily imageable (imageability effects; Balota, Cortese, SergentMarshall, Spieler, \& Yap, 2004) or that the human body can easily interact with (body-object interaction effects; Siakaluk, Pexman, Aguilera, Owen, \& Sears, 2008). These semantic richness effects are consistent with the principle that when it comes to semantic activation in lexical processing, "more is better" (Balota, Ferraro, \& Connor, 1991, p. 214).

Early research on semantic richness tended to focus on the effects of each richness dimension in isolation. More recently, advances have been made in studies with large word sets that afford the opportunity to simultaneously examine the effects of several richness variables on lexical-semantic processing. These studies have shown that several richness dimensions have unique relationships with lexical-semantic processing, and that the relative proportions of variance accounted for by particular dimensions vary across tasks (Pexman, Hargreaves, Siakaluk, Bodner, \& Pope, 2008; Yap, Pexman, Wellsby, Hargreaves, \& Huff, 2012; Yap, Tan, Pexman, \& Hargreaves, 2011). These results suggest that semantic representation may be multidimensional, and that lexical-semantic processing is dynamic, with the task demands influencing the meanings accessed. Much of this research, however, has focused on the meanings of concrete words - that is, words with tangible referents (e.g., truck; Pexman et al., 2008; Yap et al., 
2012). Thus, although we now have clues about the representation of concrete concepts, we know much less about the representation of abstract concepts (e.g., truth).

For a number of reasons, it is important that we investigate the representation of abstract concepts. First, as was noted by Recchia and Jones (2012), abstract words represent a large proportion of the words that we know, and they are overrepresented in the words used most frequently. Second, whereas the embodied-cognition framework has been successfully applied to explain conceptual processing for concrete words, abstract words have been described as a challenge for embodied accounts (e.g., Mahon \& Caramazza, 2008), but the role of embodiment in abstract meaning has not been tested extensively. Third, other theoretical suggestions have been made about what the important dimensions of abstract meaning are (e.g., Crutch, Ridha, \& Warrington, 2006), but as yet, little empirical work has tested these claims.

The purpose of the present research was to extend the investigation of semantic richness effects to abstract words, in order to provide insight into the representation of abstract meaning. We considered dimensions derived from specific proposals in the literature about the semantic representation of abstract words.

\section{Candidate semantic dimensions}

Context It has been argued that an important dimension of word meaning, and one that captures the processing differences between concrete and abstract words, is context availability (CA; i.e., participants rate words for the ease with which they evoke contexts or circumstances in which they might appear). Schwanenflugel, Harnishfeger, and Stowe (1988) demonstrated that the usual concreteness effect (faster lexical decisions for concrete than for abstract words) was eliminated when CA was controlled. Pecher, Boot, and Van Dantzig (2011) noted that CA may also tap the extent to which word meanings involve situated simulations, a construct that has been proposed to be important for abstract meaning (Barsalou, 1999; Barsalou \& Wiemer-Hastings, 2005). Lexical decisions tend to be facilitated for words with relatively high CA (e.g., Colombo \& Burani, 2002), though to our knowledge this CA effect has not been examined for abstract words alone.

Sensorimotor experience Although abstract words do not refer to concrete objects, it has been argued that their meanings can still be captured in embodied ways. Juhasz, Yap, Dicke, Taylor, and Gullick (2011) offered a broad characterization of sensory experience that they argued could be extended to all classes of words, including abstract words. They characterized a semantic dimension called sensory experience rating (SER), which they described as the extent to which words evoke sensations such as taste, touch, sight, sound, or smell. Juhasz et al. reported that lexical decisions were faster for words with higher SER ratings, but they did not examine the effect of SER on abstract words separately.

Emotion One recent proposal (Vigliocco, Meteyard, Andrews, \& Kousta, 2009) emphasized the role of emotional information as being central to the representation of abstract words. That is, emotion provides a means to ground abstract meaning in experience. Indeed, Newcombe, Cambell, Siakaluk, and Pexman (2012) reported that the semantic categorization of abstract words was facilitated for words with higher-rated emotional experience. Whereas Newcombe et al. examined emotional experience as a unitary dimension, we took a different approach here. Following the tradition in emotion research, valence (positive-negative dimension) and arousal (intensity of the corresponding feeling) were examined separately (Russell, 1980). This approach was motivated by evidence that valence and arousal have separable effects when processing word meaning. For instance, whereas valence is associated with recognition memory accuracy for single word stimuli, arousal is not (Adelman \& Estes, 2013). Furthermore, valence and arousal are associated with activity in different brain regions (Lewis, Critchley, Rotshtein, \& Dolan, 2006).

Valence is a dimension that has been shown to influence lexical processing; Estes and Adelman (2008) showed that lexical decisions were slower to negative words and faster to positive words. Kousta, Vinson, and Vigliocco (2009) showed a different effect of valence, however, with faster responses for positive and negative than for neutral words. Given these mixed findings, we examined valence both as a unitary dimension and as two separate dimensions of positivity and negativity. Arousal ratings index the degree of excitement and intensity associated with word meaning. If this kind of information is represented as part of lexical semantics, we might observe faster processing for relatively more arousing words.

Co-occurrence Another proposal has been that a word's meaning can be derived from its usage. On the basis of the lexical co-occurrence model of Burgess and Lund (2000), Buchanan et al. (2001) extracted semantic neighborhood information (words' semantic neighbors are those that are used in similar lexical contexts) and found faster lexicaldecision latencies for words with larger semantic neighborhoods (SN). In a recent study, Recchia and Jones (2012) demonstrated that high SN was associated with faster lexical decisions for abstract words.

Association A final proposal has been that associative network structure might be particularly important to abstract concepts (e.g., Crutch et al., 2006; Duñabeitia, Avilés, Afonso, Scheepers, \& Carreiras, 2009; but see Marques \& Nunes, 2012). The relative richness of this type of information 
can be indexed by a word's number of associates (NoA). Several studies have now shown that words with higher NoAs produce faster responses in lexical and semantic tasks (Balota et al., 2004; Buchanan et al., 2001; Duñabeitia et al., 2008; Pexman, Hargreaves, Edwards, Henry, \& Goodyear, 2007), but these studies have not examined NoA effects for abstract words in particular.

\section{The present study}

The purpose of our study was to examine the independence and relative contributions of six semantic richness variables to the visual word recognition of abstract nouns: (1) CA, (2) SER, (3) valence, (4) arousal, (5) SN, and (6) NoA. We first assessed the relationships between these variables, to determine whether they might tap the same (or overlapping) underlying constructs. We next examined the extents to which these variables predict unique variability in lexical decision and semantic categorization tasks (LDT and SCT, respectively).

In previous research, facilitatory effects of semantic richness have been observed for concrete words in both LDT and SCT. The explanation for the effects has varied with task. According to the feedback activation framework (e.g., Hino \& Lupker, 1996), words associated with more semantic information generate more semantic activation and, in turn, stronger feedback activation to orthographic units. In LDT, responses are based primarily on orthographic processing, and thus benefit from semantic richness indirectly, via stronger feedback. In SCT, responses are based primarily on processing in the semantic units, and greater levels of semantic activation are thought to facilitate semantic processing. As in previous research (e.g., Pexman et al., 2008; Yap et al., 2012) we expected lexical factors (frequency, orthographic characteristics) to explain relatively more variance in the LDT and less in the SCT, and semantic factors (richness dimensions) to explain relatively less variance in the LDT and more in the SCT. We further expected that the richness effects observed in an LDT would be those that can be attributed to broad and cursory semantic processing, whereas richness effects in an SCT would be those that can be attributed to more focused semantic processing shaped by the particular decision category presented.

\section{Method}

Participants

A total of 239 participants completed six rating tasks: abstractness ( $n=48$ participants), age of acquisition (AoA; $n=51)$, SER $(n=46), \mathrm{CA}(n=32)$, and arousal and valence $(n=62)$. An additional 39 participants completed the LDT, and 41 completed the SCT. All of the participants were undergraduate students at the University of Calgary who received partial course credit for participation and reported English proficiency and normal or corrected-to-normal vision.

Stimuli and procedure

Rating tasks We first selected items from the Nelson, McEvoy, and Schreiber (1998) norms with concreteness ratings below $3.5(\mathrm{QCON})$ and that were classified exclusively as nouns (Miller, 1990). We collected abstractness ratings for these 366 nouns (and 92 concrete fillers), following Paivio, Yuille, and Madigan (1968), with a slight modification: $1=$ Highly abstract, $7=$ Highly concrete. We selected 207 abstract nouns with means below 3.7 in the our ratings and collected AoA, CA, SER, arousal, and valence ratings for these items.

We adopted rating instructions from the following sources: AoA ( $1=$ age $0-2,7=$ age 13 and older $)$ from Carroll and White (1973), using the 7-point scale of Gilhooly and Hay (1977); SER ( $1=$ no sensory experience, 7 = strong sensory experience) from Juhasz et al. (2011); CA $(1=$ very hard to think of a context, $7=$ very easy to think of a context) from Schwanenflugel and Shoben (1983); and arousal $(1=$ completely excited, $5=$ completely calm $)$ and valence $(1=$ completely happy, $5=$ completely unhappy $)$ from Bradley and Lang (1999). Participants completed each rating task in an online survey.

We extracted words' NoAs from Nelson et al. (1998), their SN information from Wordmine2 (global semantic neighborhood size; Durda \& Buchanan, 2006), and all

Table 1 Stimulus characteristics and mean lexical decision (LDT) and semantic categorization (SCT) response latencies (RTs, in milliseconds)

\begin{tabular}{lll}
\hline Variable $(n=207)$ & $M$ & $S D$ \\
\hline Log frequency (SUBTL; Brysbaert \& New, 2009) & 2.54 & 0.65 \\
Orthographic Levenshtein distance (Yarkoni et & 2.64 & 0.80 \\
al., 2008) & & \\
Age of acquisition $^{*}$ & 4.84 & 0.83 \\
Context availability $^{*}$ & 5.61 & 0.48 \\
Sensory experience rating $^{*}$ & 2.82 & 0.55 \\
Valence $^{*}$ & 2.71 & 0.91 \\
Arousal $^{*}$ & 2.76 & 0.35 \\
Semantic neighborhood (Durda \& Buchanan, $^{2006)}$ & 13.99 & 48.01 \\
Number of associates (Nelson et al., 1998) & 17.18 & 5.00 \\
LDT RTs & 668.85 & 210.27 \\
SCT RTs & 759.36 & 227.95 \\
\hline
\end{tabular}

${ }^{*}$ Ratings collected from University of Calgary participants. 
Table 2 Correlations between predictor variables and dependent measures

\begin{tabular}{|c|c|c|c|c|c|c|c|c|c|c|c|}
\hline Variable & 1 & 2 & 3 & 4 & 5 & 6 & 7 & 8 & 9 & 10 & 11 \\
\hline 1. Log frequency & - & & & & & & & & & & \\
\hline 2. OLD & $-.35^{* * *}$ & - & & & & & & & & & \\
\hline 3. AoA & $-.68^{* * *}$ & $.21^{* *}$ & - & & & & & & & & \\
\hline 4. $\mathrm{CA}$ & $.58^{* * *}$ & -.11 & $-.74^{* * *}$ & - & & & & & & & \\
\hline 5. SER & $.44^{* * *}$ & -.09 & $-.51^{* * *}$ & $.62^{* * *}$ & - & & & & & & \\
\hline 6. Valence & $-.21^{* *}$ & -.08 & $.27^{* * *}$ & -.11 & -.05 & - & & & & & \\
\hline 7. Arousal & -.13 & -.04 & $.22^{* *}$ & $-.30^{* * *}$ & $-.49^{* * *}$ & $-.21^{* *}$ & - & & & & \\
\hline 8. $\mathrm{SN}$ & $.35^{* * *}$ & $-.16^{*}$ & $-.23^{* *}$ & .05 & -.02 & -.08 & -.05 & - & & & \\
\hline 9. NoA & -.01 & .09 & .06 & .05 & .00 & .06 & .01 & -.05 & - & & \\
\hline 10. LDT RT ( $z$ score) & $-.73^{* * *}$ & $.41^{* * *}$ & $.61^{* * *}$ & $-.62^{* * *}$ & $-.46^{* *}$ & $.15^{*}$ & $.17^{*}$ & $-.18^{* *}$ & .00 & - & \\
\hline 11. SCT RT ( $z$ score) & $-.33^{* * *}$ & .06 & $.23^{* *}$ & $-.29^{* * *}$ & $-.34^{* * *}$ & $.22^{* *}$ & .09 & -.08 & -.04 & $.49^{* * *}$ & - \\
\hline
\end{tabular}

Log frequency, SUBTL word frequency; OLD, orthographic Levenshtein distance; AoA, age of acquisition; CA, context availability; SER, sensory experience rating; SN, semantic neighborhood; NoA, number of associates; LDT, lexical decision task; SCT, semantic categorization task. ${ }^{*} p<.05 ;{ }^{* *} p<.01 ;{ }^{* * *} p<.001$.

other variables from the English Lexicon Project (Balota et al., 2007).

LDT and SCT We collected LDT and SCT data using the following procedure: Testing began with practice trials with verbal feedback. On each trial, an item was presented in the center of a 20 -in. monitor. Each trial began with a 1,000-ms fixation point, which was replaced by a stimulus item. The 207 abstract nouns were presented with 207 nonwords (matched on length) in the LDT, and with 207 concrete nouns (matched on length) in the SCT. Participants were instructed to make their responses by pressing the far right button on a response box if the item was a word (LDT) or an abstract word (SCT), and otherwise to withhold a response. We used a go/no-go procedure in order to encourage participants to focus on abstractness (and not the absence of concreteness) as the decision category in SCT; we used a go/no-go procedure in the LDT to facilitate comparisons between the tasks.

\section{Results}

Rating tasks

Descriptive statistics for all variables are presented in Table 1. Zero-order correlations (Table 2) showed that the semantic richness variables tended to be only modestly correlated; the strongest relationship was between CA and SER, at $r=.62$ : Words rated as easily evoking contexts also tended to be associated with more sensory experience. In general, these relationships suggest that the richness variables do not tap the same underlying construct. We next examined how these variables were related to standardized LDT and SCT responses (standardized decision latencies minimize the influence of participant processing speed and variability; Faust, Balota, Spieler, \& Ferraro, 1999). We used item-wise hierarchical regression analyses, with LDT and (separately) SCT responses as dependent variables, and in the first step included as predictor variables a number of dimensions that influence lexical processing: frequency (log-transformed SUBTL frequency; Brysbaert \& New, 2009), orthographic characteristics (orthographic Levenshtein distance; Yarkoni, Balota, \& Yap, 2008), and AoA. We treated AoA as a control variable, rather than a semantic richness variable, because there is some disagreement as to whether AoA is a lexical or a lexical-semantic variable (e.g., Cortese \& Khanna, 2007; Zevin \& Seidenberg, 2002). In

Table 3 Standardized response time (RT) regression coefficients from Steps 1 and 2 of the item-level regression analyses for the lexical decision (LDT) and semantic categorization (SCT) tasks

\begin{tabular}{lll}
\hline Variable & LDT $(n=206$ nouns $)$ & SCT $(n=202$ nouns $)$ \\
\hline Step 1: Control Variables & \\
Log Frequency & $-.51^{* * *}$ & $-.35^{* * *}$ \\
OLD & $.18^{* * *}$ & -.07 \\
AoA & $.22^{* * *}$ & -.01 \\
Adjusted $R^{2}$ & $.57^{* * *}$ & $.09^{* * *}$ \\
Step 2: Semantic Richness Variables & \\
CA & $-.29^{* * *}$ & -.11 \\
SER & -.04 & $-.26^{* *}$ \\
Valence & .03 & $.19^{* * *}$ \\
Arousal & .01 & -.00 \\
SN & .04 & -.03 \\
NoA & -.01 & -.04 \\
Adjusted $R^{2}$ & $.61^{* * *}$ & $.16^{* * *}$ \\
Change in $R^{2}$ & $.05^{* * *}$ & $.09^{* * *}$ \\
\hline${ }^{*} p<.05 ;{ }^{* *} p<.01 ;$ & ${ }^{* * * *} p<.001$.
\end{tabular}


the second analytic step, we examined the contributions of each of the six semantic richness variables: CA, SER, valence, arousal, SN, and NoA.

Latencies were excluded from the analyses for participants (one in LDT, two in SCT) or items (one in LDT, five in SCT) with accuracy less than $70 \%$. In addition, incorrect trials ( $2 \%$ in LDT, $4.38 \%$ in SCT) and trials with latencies more than three standard deviations from the participant's mean (1.97\% in LDT, $1.99 \%$ in SCT) were also excluded.

The regression results for response latencies are presented in Table 3 (too few response errors were committed to warrant analyses). As is illustrated in Table 3, we observed one significant semantic richness effect in the LDT: faster LDT latencies for words that more easily evoke contextual information. In the SCT, we observed two richness effects: faster latencies for words associated with either richer sensory or more positive emotional experience. ${ }^{1}$

We conducted a second set of regression analyses in order to investigate the effects of valence when this dimension was recoded as two separate constructs: positivity (scores from the neutral midpoint to the positive end of the valence scale) and negativity (scores from the neutral midpoint to the negative end of the valence scale). The results showed that neither of these variables had a significant relationship with LDT latencies, and that in the SCT positivity was associated with faster latencies $(p<.05)$, whereas negativity was associated with slower latencies $(p<.05)$. As such, these results corroborate the inferences drawn from the first analyses, in which valence was treated as a single, bipolar dimension.

\section{Discussion}

The aim of our study was to investigate the influence of semantic richness variables on the lexical-semantic processing of abstract words. The results demonstrated that several semantic richness dimensions are related to this type of processing: Abstract words that evoke relatively more contextual information were processed more quickly in the LDT, whereas abstract words that evoke relatively more

\footnotetext{
${ }^{1}$ Given the strong relationships between AoA and two of our richness variables (SER and CA), we evaluated the variance inflation factors (VIFs) and found values of 5.07 for the control variables and 9.92 for the richness variables in the LDT, and of 5.05 and 9.83 for the respective variables in the SCT, suggesting that multicollinearity could be an issue. When we reran the regression analyses with AoA removed, however, the pattern of results did not change: $\mathrm{CA}$ was significant (more contexts= faster latencies) in the LDT analysis, and SER (more sensory experience $=$ faster latencies) and valence (more positive $=$ faster latencies) were significant in the SCT analysis.
}

sensory experience and positive emotion were processed more quickly in the SCT.

The present results are broadly compatible with proposals that the representation of word meaning involves multiple systems; in particular, linguistic and perceptual systems (Dove, 2011; Louwerse \& Jeuniaux, 2010; Paivio, 1986, 2007). For instance, the language-andsituated-simulation theory (Barsalou, Santos, Simmons, \& Wilson, 2008; Simmons, Hamann, Harenski, Hu, \& Barsalou, 2008) holds that both the language and modal systems are important for conceptual representation (see also Andrews, Vigliocco, \& Vinson, 2009). The language system includes information about linguistic form and the associations between words. The modal simulation system includes information about the sensorimotor states activated by interactions with a word's referents. The relative influence of each system will depend, in part, on the task demands (Louwerse \& Jeuniaux, 2010). Although most tasks recruit both systems, in a task that can be performed primarily on the basis of evaluation of linguistic form, the language system will be influential, whereas in a task that involves deeper consideration of meaning, the modal simulation system will be relatively more influential. Our results are compatible with this dynamic, embodied framework. Since CA, SER, and valence could all be construed as dimensions of simulation, and because the effects of these variables dissociate across tasks, one would further need to assume that the simulation system is multidimensional. That is, CA, SER, and valence reflect different (nonunitary) aspects of simulated meaning.

Our results suggest that the semantic processing invoked in completing the LDT taps words' situational histories, whereas the SCT taps the sensory and emotional aspects of words' meanings. Participants construct different semantic representations as a function of task demands, so it is certainly possible that other semantic richness dimensions could be important in different tasks or with different item sets. The valence effect observed here involved relatively faster latencies for positive words, and slower latencies for negative words. This suggests that participants access valence information to respond in the SCT and that valence is an important aspect of abstract meaning (Vigliocco et al., 2009). Why positive valence facilitates "abstractness" decisions in the SCT is a matter that will need to be explored more thoroughly in future research.

In summary, the semantic richness approach holds promise as a strategy for addressing the long-standing problem of representation for abstract word meaning; abstract meaning may be grounded in situations, emotions, and sensory experience.

Author note This work was supported in part by a Natural Sciences and Engineering Research Council (NSERC) of Canada Discovery Grant to P.M.P. 


\section{References}

Adelman, J. S., \& Estes, Z. (2013). Emotion and memory: A recognition advantage for positive and negative words independent of arousal. Manuscript under review.

Andrews, M., Vigliocco, G., \& Vinson, D. (2009). Integrating experiential and distributional data to learn semantic representations. Psychological Review, 116, 463-498. doi:10.1037/a0016261

Balota, D. A., Cortese, M. J., Sergent-Marshall, S. D., Spieler, D. H., \& Yap, M. J. (2004). Visual word recognition of single-syllable words. Journal of Experimental Psychology: General, 133, 283-316. doi:10.1037/0096-3445.133.2.283

Balota, D. A., Ferraro, F. R., \& Connor, L. T. (1991). On the early influence of meaning in word recognition: A review of the literature. In P. J. Schwanenflugel (Ed.), The psychology of word meanings (pp. 187-222). Hillsdale, NJ: Erlbaum.

Balota, D. A., Yap, M. J., Cortese, M. J., Hutchison, K. A., Kessler, B., Loftis, B., . . . Treiman, R. (2007). The English Lexicon Project. Behavior Research Methods, 39, 445-459. doi:10.3758/BF03193014

Barsalou, L. W. (1999). Perceptual symbol systems. Behavioral and Brain Sciences, 22, 577-609, disc. 609-660. doi:10.1017/ S0140525X99002149

Barsalou, L. W., Santos, A., Simmons, W. K., \& Wilson, C. D. (2008). Language and simulation in conceptual processing. In M. De Vega, A. M. Glenberg, \& A. C. Graesser (Eds), Symbols and embodiment: Debates on meaning and cognition (pp. 245-283). Oxford, UK: Oxford University Press.

Barsalou, L. W., \& Wiemer-Hastings, K. (2005). Situating abstract concepts. In D. Pecher \& R. Zwaan (Eds.), Grounding cognition: The role of perception and action in memory, language, and thought (pp. 129-163). Cambridge, UK: Cambridge University Press.

Bradley, M. M., \& Lang, P. J. (1999). Affective norms for English words (ANEW): Stimuli, instruction manual and affective ratings (Technical Report No. C-1). Gainesville, FL: University of Florida, NIMH Center for Research in Psychophysiology.

Brysbaert, M., \& New, B. (2009). Moving beyond Kučera \& Francis: A critical evaluation of current word frequency norms and the introduction of a new and improved word frequency measure for American English. Behavior Research Methods, 41, 977-990. doi:10.3758/BRM.41.4.977

Buchanan, L., Westbury, C., \& Burgess, C. (2001). Characterizing semantic space: Neighborhood effects in word recognition. Psychonomic Bulletin \& Review, 8, 531-544.

Burgess, C., \& Lund, K. (2000). The dynamics of meaning in memory. In E. Dietrich \& A. B. Markman (Eds.), Cognitive dynamics: Conceptual and representational change in humans and machines (pp. 117-156). Mahwah, NJ: Erlbaum.

Carroll, J. B., \& White, M. N. (1973). Word frequency and age of acquisition as determiners of picture-naming latency. Quarterly Journal of Experimental Psychology, 25, 85-95. doi:10.1080/ 14640747308400325

Colombo, L., \& Burani, C. (2002). The influence of age of acquisition, root frequency, and context availability in processing nouns and verbs. Brain \& Language, 81, 398-411.

Cortese, M. J., \& Khanna, M. M. (2007). Age of acquisition predicts naming and lexical-decision performance above and beyond 22 other predictor variables: An analysis of 2,342 words. Quarterly Journal of Experimental Psychology, 60, 1072-1082. doi:10.1080/ 17470210701315467

Crutch, S. J., Ridha, B. H., \& Warrington, E. K. (2006). The different frameworks underlying abstract and concrete knowledge: Evidence from a bilingual patient with a semantic refractory access dysphasia. Neurocase, 12, 151-163.

Dove, G. (2011). On the need for embodied and dis-embodied cognition. Frontiers in Psychology, 1:242. doi:10.3389/fpsyg.2010.00242
Duñabeitia, J. A., Avilés, A., Afonso, O., Scheepers, C., \& Carreiras, M. (2009). Qualitative differences in the representation of abstract versus concrete words: Evidence from the visual-world paradigm. Cognition, 110, 284-292. doi:10.1016/j.cognition.2008.11.012

Duñabeitia, J. A., Avilés, A., \& Carreiras, M. (2008). NoA's ark: Influence of the number of associates in visual word recognition. Psychonomic Bulletin \& Review, 15, 1072-1077. doi:10.3758/PBR.15.6.1072

Durda, K., \& Buchanan, L. (2006). WordMine2 [Online]. Available at http://web2.uwindsor.ca/wordmine

Estes, Z., \& Adelman, J. S. (2008). Automatic vigilance for negative words is categorical and general. Emotion, 8, 453-457. doi:10.1037/a0012887

Faust, M. E., Balota, D. A., Spieler, D. H., \& Ferraro, F. R. (1999). Individual differences in information-processing rate and amount: Implications for group differences in response latency. Psychological Bulletin, 125, 777-799.

Gilhooly, K. J., \& Hay, D. (1977). Imagery, concreteness, age-ofacquisition, familiarity, and meaningfulness values of 205 fiveletter words having single-solution anagrams. Behavior Research Methods \& Instrumentation, 9, 12-17.

Grondin, R., Lupker, S. J., \& McRae, K. (2009). Shared features dominate semantic richness effects for concrete concepts. Journal of Memory and Language, 60, 1-19. doi:10.1016/j.jml.2008.09.001

Hino, Y., \& Lupker, S. J. (1996). Effects of polysemy in lexical decision and naming: An alternative to lexical access accounts. Journal of Experimental Psychology: Human Perception and Performance, 22, 1331-1356. doi:10.1037/0096-1523.22.6.1331

Juhasz, B. J., Yap, M. J., Dicke, J., Taylor, S. C., \& Gullick, M. M. (2011). Tangible words are recognized faster: The grounding of meaning in sensory and perceptual systems. Quarterly Journal of Experimental Psychology, 64, 1683-1691. doi:10.1080/17470218.2011.605150

Kousta, S. T., Vinson, D. P., \& Vigliocco, G. (2009). Emotion words, regardless of polarity, have a processing advantage over neutral words. Cognition, 112, 473-481. doi:10.1016/j.cognition.2009.06.007

Lewis, P. A., Critchley, H. D., Rotshtein, P., \& Dolan, R. J. (2006). Neural correlates of processing valence and arousal in affective words. Cerebral Cortex, 17, 742-748.

Louwerse, M. M., \& Jeuniaux, P. (2010). The linguistic and embodied nature of conceptual processing. Cognition, 114, 96-104.

Mahon, B. Z., \& Caramazza, A. (2008). A critical look at the embodied cognition hypothesis and a new proposal for grounding conceptual content. Journal of Physiology, 102, 59-70. doi:10.1016/ j.jphysparis.2008.03.004

Marques, J. F., \& Nunes, L. D. (2012). The contributions of language and experience to the representation of abstract and concrete words: Different weights but similar organizations. Memory \& Cognition, 40, 1266-1275. doi:10.3758/s13421-012-0220-6

McRae, K., Cree, G. S., Seidenberg, M. S., \& McNorgan, C. (2005). Semantic feature production norms for a large set of living and nonliving things. Behavior Research Methods, 37, 547-559. doi:10.3758/BF03192726

Miller, G. A. (1990). WordNet: An online lexical database. International Journal of Lexicography, 3, 235-312.

Nelson, D. L., McEvoy, C. L., \& Schreiber, T. A. (1998). The University of South Florida word association, rhyme, and word fragment norms. Retrieved March 9, 2011, from http://w3.usf.edu/FreeAssociation/

Newcombe, P. I., Cambell, C., Siakaluk, P. D., \& Pexman, P. M. (2012). Effects of emotional and sensorimotor knowledge in semantic processing of concrete and abstract nouns. Frontiers in Human Neuroscience, 6:113. doi:10.3389/fnhum.2012.00113

Paivio, A. (1986). Mental representations: A dual coding approach. New York, NY: Oxford University Press.

Paivio, A. (2007). Mind and its evolution: A dual coding theoretical approach. Mahwah, NJ: Erlbaum.

Paivio, A., Yuille, J. C., \& Madigan, S. A. (1968). Concreteness, imagery, and meaningfulness values for 925 nouns. Journal of 
Experimental Psychology, 76(1, Pt. 2), 1-25. doi:10.1037/ h0025327

Pecher, D., Boot, I., \& Van Dantzig, S. (2011). Abstract concepts: Sensory-motor grounding, metaphors, and beyond. Psychology of Learning and Motivation, 54, 217-248.

Pexman, P. M. (2012). Meaning-level influences on visual word recognition. In J. S. Adelman (Ed.), Visual word recognition: Vol. 2. Meaning and context, individuals and development (pp. 24-43). Hove, UK: Psychology Press.

Pexman, P. M., Hargreaves, I. S., Edwards, J. D., Henry, L. C., \& Goodyear, B. G. (2007). The neural consequences of semantic richness: When more comes to mind, less activation is observed. Psychological Science, 18, 401-406.

Pexman, P. M., Hargreaves, I. S., Siakaluk, P. D., Bodner, G. E., \& Pope, J. (2008). There are many ways to be rich: Effects of three measures of semantic richness on visual word recognition. Psychonomic Bulletin \& Review, 15, 161-167. doi:10.3758/PBR.15.1.161

Pexman, P. M., Lupker, S. J., \& Hino, Y. (2002). The impact of feedback semantics in visual word recognition: Number-of-features effects in lexical decision and naming tasks. Psychonomic Bulletin \& Review, 9, 542-549. doi:10.3758/BF03196311

Recchia, G., \& Jones, M. N. (2012). The semantic richness of abstract concepts. Frontiers in Human Neuroscience, 6:315. doi:10.3389/ fnhum.2012.00315

Russell, J. A. (1980). A circumplex model of affect. Journal of Personality and Social Psychology, 39, 1161-1178.

Schwanenflugel, P. J., Harnishfeger, K. K., \& Stowe, R. W. (1988). Context availability and lexical decisions for abstract and concrete words. Journal of Memory and Language, 27, 499-520.
Schwanenflugel, P. J., \& Shoben, E. J. (1983). Differential context effects in the comprehension of abstract and concrete verbal materials. Journal of Experimental Psychology: Learning, Memory, and Cognition, 9, 82-102. doi:10.1037/0278-7393.9.1.82

Siakaluk, P. D., Pexman, P. M., Aguilera, L., Owen, W. J., \& Sears, C. R. (2008). Evidence for the activation of sensorimotor information during visual word recognition: The body-object interaction effect. Cognition, 106, 433-443. doi:10.1016/j.cognition. 2006.12.011

Simmons, W. K., Hamann, S. B., Harenski, C. L., Hu, X. P., \& Barsalou, L. W. (2008). fMRI evidence for word association and situated simulation in conceptual processing. Journal of Physiology, 102, 106-119.

Vigliocco, G., Meteyard, L., Andrews, M., \& Kousta, S. (2009). Toward a theory of semantic representation. Language \& Cognition, 1, 219-248.

Yap, M. J., Pexman, P. M., Wellsby, M., Hargraeves, I. S., \& Huff, M. (2012). An abundance of riches: Cross task comparisons of semantic richness effects in visual word recognition. Frontiers in Human Neuroscience, 6:53. doi:10.3389/fnhum.2012.00053

Yap, M. J., Tan, S. E., Pexman, P. M., \& Hargreaves, I. S. (2011). Is more always better? Effects of semantic richness on lexical decision, speeded pronunciation, and semantic classification. Psychonomic Bulletin \& Review, 18, 742-750. doi:10.3758/s13423-011-0092-y

Yarkoni, T., Balota, D. A., \& Yap, M. J. (2008). Moving beyond Coltheart's $N$ : A new measure of orthographic similarity. Psychonomic Bulletin \& Review, 15, 971-979. doi:10.3758/PBR.15.5.971

Zevin, J. D., \& Seidenberg, M. S. (2002). Age of acquisition effects in word reading and other tasks. Journal of Memory and Language, 47, 1-29. doi:10.1006/jmla.2001.2834 